\title{
Three-dimensional image sensing, encryption, compression, and transmission using digital holography
}

\author{
Thomas J. Naughton, ${ }^{a}$ Enrique Tajahuerce, ${ }^{b}$ John B. McDonald, ${ }^{a}$ and Bahram Javidi ${ }^{c}$ \\ ${ }^{a}$ Department of Computer Science, National University of Ireland/Maynooth, Maynooth, \\ County Kildare, Ireland \\ ${ }^{b}$ Departament de Ciències Experimentals, Universitat Jaume I, Av. Vicent Sos Baynat, s/n, \\ 12071 Castelló, Spain \\ ${ }^{c}$ Electrical and Computer Engineering Department, University of Connecticut, 371 Fairfield \\ Road, Unit 2157, Storrs, CT 06269-2157, USA
}

\begin{abstract}
We present the results of applying data compression techniques to encrypted three-dimensional (3D) objects. The objects are captured using phase-shift digital holography and encrypted using a random phase mask in the Fresnel domain. Both the amplitude and the phase of our 3D objects are encrypted using this technique. The advantage of a digital representation of the optical wavefronts is that they can be processed and transmitted using conventional means. Arbitrary views of the 3D objects are decrypted and reconstructed using digital propagation. Compression is applied to the encrypted digital holograms prior to transmission. Degradation due to lossy quantization compression is measured in the reconstruction domain. Finally, we use a speedup metric to validate that our compression techniques are viable for time-critical 3D imaging applications. Our techniques are suitable for a range of secure 3D object storage and transmission applications.
\end{abstract}

Keywords: three-dimensional image processing, digital holography, optical image encryption, image compression, optical information processing

\section{INTRODUCTION}

Digital holography ${ }^{1-10}$ is a variant of regular optical holography that provides holograms in digital form by recording interference patterns with a CCD detector. It avoids analog recording and corresponding chemical development, and allows us to record, process, and transmit phase and amplitude information using conventional digital means. To record the digital hologram of a three-dimensional (3D) object we use a phase-shift interferometer. ${ }^{8-10}$ Arbitrary views of the $3 \mathrm{D}$ objects can be reconstructed digitally or optically. ${ }^{11,12}$ We have extended these techniques for security applications, such as recognition, encryption, and authentication of 3D information. ${ }^{9-19}$

In this paper we present the results of applying data compression techniques to optically encrypted 3D objects. Optical image encryption has received much attention recently, ${ }^{11,16,19-36}$ and is often performed with a random phase mask positioned in the input, Fresnel, or Fourier domains, or combination of domains. These invariably produce a complex-valued encrypted image. Our digital holograms are encrypted using a random phase mask in the Fresnel domain, and are captured using phase-shift interferometry (PSI), as explained in Sect. 2. Both the amplitude and the phase of our 3D objects are encrypted using this technique. Compression is then applied to the encrypted digital holograms for more efficient transmission of the 3D objects. Lossless techniques, on their own, perform very poorly on encrypted (and unencrypted) digital holograms because of their white noise characteristics. ${ }^{17,19}$ In Sect. 3, we describe the use of lossy quantization compression to reduce the amount of information in each complex-valued holographic pixel. Treatments of quantization in holograms can be found in the literature, ${ }^{37,38}$ and compression of real-valued ${ }^{39}$ and complex-valued ${ }^{17-19}$ digital holograms has received some attention to date. Degradation due to lossy quantization compression is measured in the reconstruction

Further author information: TN: tom.naughton@may.ie; ET: enrique.tajahuerce@uji.es; JMcD: johnmcd@cs.may.ie; BJ: bahram@engr.uconn.edu 


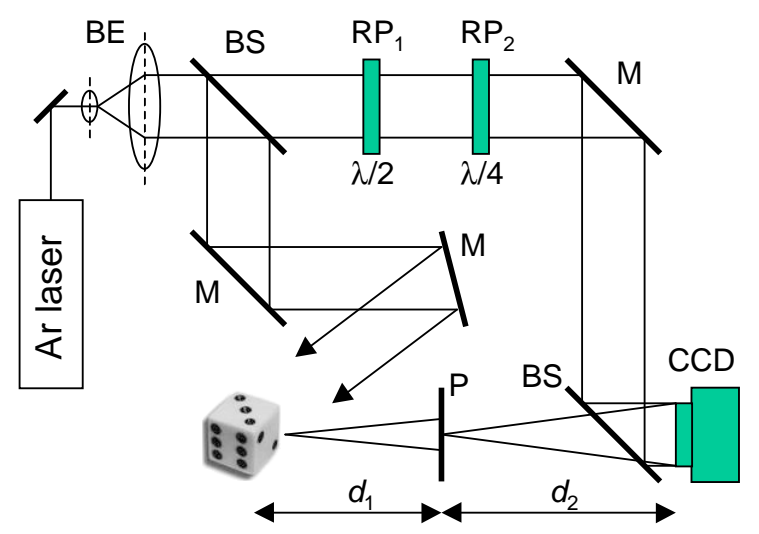

Figure 1. Experimental setup for 3D object encryption using phase-shift digital holography: BE, beam expander; BS, beam splitter; M, mirror; RP, retardation plate; $\mathrm{P}$, phase mask

domain. This involves comparing the reconstruction from a compressed and decompressed digital hologram with the reconstruction from the original digital hologram. However, in order to be useful for a real-time 3D object capture, transmission, and reconstruction system, the compression strategies must be shown to admit efficient algorithms that make it advantageous to spend time compressing and decompressing rather than simply transmitting the original data. To this end, in Sect. 4 we define a speedup metric that combines space gains due to compression with temporal overheads due to the compression routine and transmission serialization. We empirically verify transmission speedup due to compression, in Sect. 5, using a special-purpose Internet-based networking application. Finally, we conclude in Sect. 6.

\section{ENCRYPTION AND DECRYPTION}

The encrypted complex-valued holograms are captured using an optical setup (shown in Fig. 1) based on a MachZehnder interferometer architecture. ${ }^{9,10}$ A linearly polarized Argon ion $(\lambda=514.5 \mathrm{~nm})$ laser beam is divided into object and reference beams, both of which are spatially filtered and expanded. The first beam illuminates the $3 \mathrm{D}$ object placed at a distance $d_{1}+d_{2}$ from a 10 bit $2028 \times 2044$ pixel Kodak Megaplus CCD camera. A random phase mask is placed a distance $d_{1}$ from the 3D object. Due to free-space propagation, and under the Fresnel approximation, ${ }^{40,41}$ the signal at the detector plane $H_{\mathrm{E}}(x, y)$ is given by the superposition integral

$$
\begin{aligned}
H_{\mathrm{E}}(x, y) & =\frac{-\mathrm{i}}{\lambda d_{2}} \exp \left(\mathrm{i} \frac{2 \pi}{\lambda} d_{2}\right) \iint_{-\infty}^{\infty} \exp \left[\mathrm{i} \Phi\left(x^{\prime}, y^{\prime}\right)\right] \\
& \times A_{\mathrm{M}}\left(x^{\prime}, y^{\prime}\right) \exp \left[\mathrm{i} \phi_{\mathrm{M}}\left(x^{\prime}, y^{\prime}\right)\right] \\
& \times \exp \left\{\mathrm{i} \frac{\pi}{\lambda d_{2}}\left[\left(x-x^{\prime}\right)^{2}+\left(y-y^{\prime}\right)^{2}\right]\right\} \mathrm{d} x^{\prime} \mathrm{d} y^{\prime},
\end{aligned}
$$

where $A_{\mathrm{M}}$ and $\phi_{\mathrm{M}}$ are the amplitude and phase, respectively, of the signal in the plane of, but immediately before, the random phase mask $\Phi . H_{\mathrm{E}}(x, y)$ will have both its amplitude and phase modulated by the mask and will have a dynamic range suitable for capture by a CCD camera.

The reference beam passes through half-wave plate $\mathrm{RP}_{1}$ and quarter-wave plate $\mathrm{RP}_{2}$. This linearly polarized beam can be phase-modulated by rotating the two retardation plates. Through permutation of the fast and slow axes of the plates we can achieve phase shifts of $0,-\pi / 2,-\pi$, and $-3 \pi / 2$. The reference beam combines with the light diffracted from the object and forms an interference pattern in the plane of the camera. At each of the four phase shifts we record an interferogram. Using these four intensity images, the complex-valued camera-plane wavefront can be approximated in a computer to good accuracy using PSI.

Digital holograms of five reasonably diffuse 3D objects were used in the experiments. ${ }^{17}$ Figure 2(a) shows one of the objects. This bolt object had approximate dimensions of $5 \mathrm{~mm} \times 5 \mathrm{~mm} \times 5 \mathrm{~mm}$, and was positioned $d_{1}+d_{2}=390 \mathrm{~mm}$ from the $2028 \times 2044$ pixel camera. The intensity image in Fig. $2(\mathrm{a})$ is reconstructed from a 


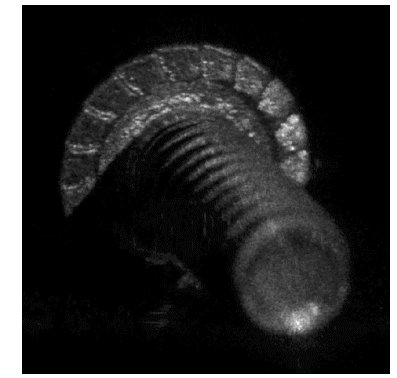

(a)

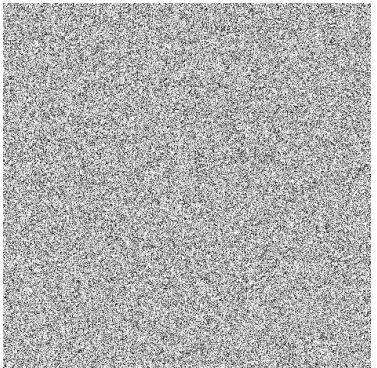

(b)

Figure 2. The bolt object (a), and an example of a random phase mask (b), used in the study

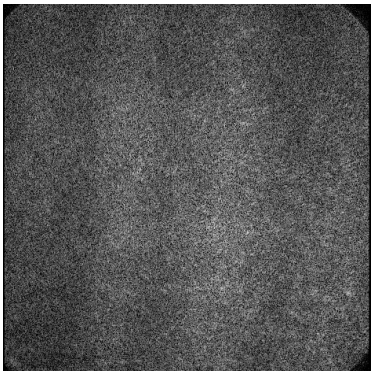

(a)

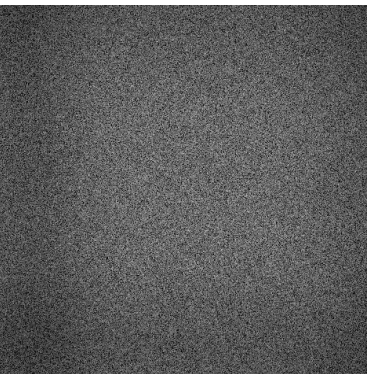

(c)

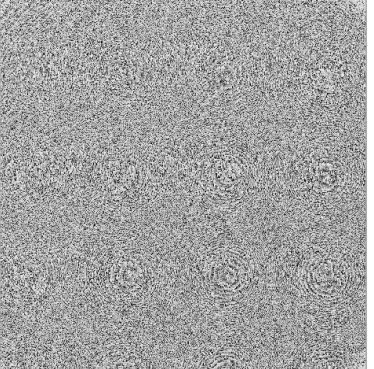

(b)

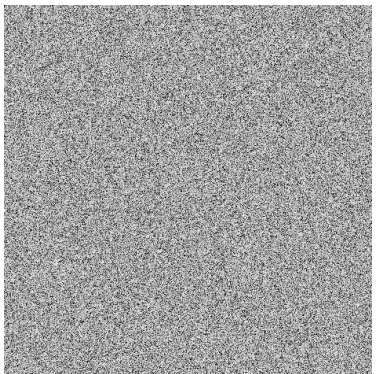

(d)

Figure 3. The bolt hologram before and after encryption: (a) amplitude and (b) phase of the original hologram, and (c) amplitude and (d) phase of the encrypted hologram

digital hologram captured using a version of the apparatus shown in Fig. 1 that did not contain a random phase mask. ${ }^{9,10}$ These reconstructions serve as a ground truth data when quantifying lossy compression errors later in the paper.

In our experiments we use digital holograms that have been captured optically using the apparatus in Fig. 1 without the phase mask, ${ }^{9,10}$ and we encrypt them on a computer using simulated free-space propagation. ${ }^{19}$ The phase mask used in the simulations is shown in Fig. 2(b). It consists of values chosen with uniform probability from the range $[0,2 \pi)$ using a pseudo-random number generator. The mask has dimensions $2048 \times 2048$ pixels and in the encryption experiments our digital holograms were enlarged from $2028 \times 2044$ pixels to these dimensions by padding with zeros. For our experiments, the mask was positioned as shown in Fig. 1 such that the ratio of the distances $d_{1}: d_{2}$ was $35: 65$. In Fig. 3 we show the amplitude and phase of the bolt hologram before encryption, and after encryption.

\section{COMPRESSION}

The loss in reconstruction quality due to applying quantization to encrypted holograms was investigated. A combined rescale and quantization step was employed. The encrypted holograms were rescaled linearly to the square in the complex plane $[-1-\mathrm{i}, 1+\mathrm{i}]$, and the real and imaginary components of each holographic pixel 
were then quantized. We choose an odd number of quantization values (or levels) for each real and imaginary value: zero, and an equal number of positive and negative levels. As a result $b$ bits encodes $\left(2^{b}-1\right)$ levels. For example, two bits encode levels $\{-1,0,1\}$, three bits encode levels $\{-1,-2 / 3,-1 / 3,0,1 / 3,2 / 3,1\}$, and so on. The combined rescale and quantization operation is defined for individual pixels as

$$
H^{\prime}(x, y)=\text { round }\left[H(x, y) \times \sigma^{-1} \times \beta\right] \times \beta^{-1}
$$

and was applied to each pixel $(x, y)$ in the encrypted hologram $H$, where

$$
\begin{aligned}
\sigma=\max \{ & |\min [\operatorname{Im}(H)]|,|\max [\operatorname{Im}(H)]|, \\
& |\min [\operatorname{Re}(H)]|,|\max [\operatorname{Re}(H)]|\},
\end{aligned}
$$

and where $\beta=2^{(b-1)}-1$. Here, $b$ represents the number of bits per real and imaginary value, $\max (\cdot) \operatorname{returns}$ the maximum scalar in its argument(s), and $\operatorname{round}(\alpha)$ is defined as $\lfloor\alpha+0.5\rfloor$. After quantization, each real and imaginary value will be in the range $[-1,1]$.

The procedure for quantifying reconstruction loss due to quantization was as follows. An encrypted digital hologram $H(x, y)$ was quantized as $H^{\prime}(x, y)$ according to Eq. (2). The hologram was decrypted and the entire hologram reconstructed, both simulated using a computer. The quality of the reconstruction $U^{\prime}(x, y)$ was calculated by a comparison with the reconstruction $U_{0}(x, y)$ from an unencrypted (and unquantized) version of the digital hologram. The two reconstructions were compared in terms of normalized rms (NRMS) difference of their intensities, defined as

$$
\begin{aligned}
D\left(U^{\prime}\right)= & \left(\sum_{m=0}^{N_{x}-1} \sum_{n=0}^{N_{y}-1}\left[\left|U_{0}(m, n)\right|^{2}-\left|U^{\prime}(m, n)\right|^{2}\right]^{2}\right. \\
& \left.\times\left\{\sum_{m=0}^{N_{x}-1} \sum_{n=0}^{N_{y}-1}\left[\left|U_{0}(m, n)\right|^{2}\right]^{2}\right\}^{-1}\right)
\end{aligned}
$$

where $(m, n)$ are discrete spatial coordinates in the reconstruction plane, and $N_{y}$ and $N_{x}$ are the height and width of the reconstructions, respectively. The slightest change to a digital hologram can result in an entirely different speckle pattern in the reconstruction domain. It could be argued that one should attempt to remove this somewhat quantization-invariant speckle effect before measuring the quantization error. Therefore, we also present the results of applying a mean filtering operation to both the original and quantized intensities prior to calculating NRMS.

Figure 4 shows a plot of NRMS difference against number of bits per (real and imaginary) data value in the encrypted hologram of the bolt object, with and without mean filtering over a neighborhood of $5 \times 5$ pixels. Figure 5 shows decrypted and reconstructed object intensities for the bolt object for selected quantization resolutions. To some degree, the quantization noise is masked visually by the presence of speckle; this would not be the case if incoherent images were encrypted and quantized.

\section{TRANSMISSION}

We can evaluate compression algorithms in terms of both space and time resource usage by using a measure called speedup. Speedup $s$ is defined as $s=P_{\mathrm{u}} / P_{\mathrm{C}}$ where $P_{\mathrm{u}}$ is the time required to process and transmit the uncompressed digital hologram and $P_{\mathrm{C}}$ is the time required to process and transmit the compressed field. If the compression strategy is useful, the processing time devoted to compression should be less than the resulting reduction in transmission time. In order to measure speedup of a compression system that resides over a public wide-area network we have found that the following three requirements should be met. Firstly, due to the temporal fluctuations in bandwidth over wide-area networks, it is necessary to average over a large number of timing measurements. Secondly, in order to accurately measure compression and decompression times, the compression routines should be removed from their controlled prototyping environment and executed 


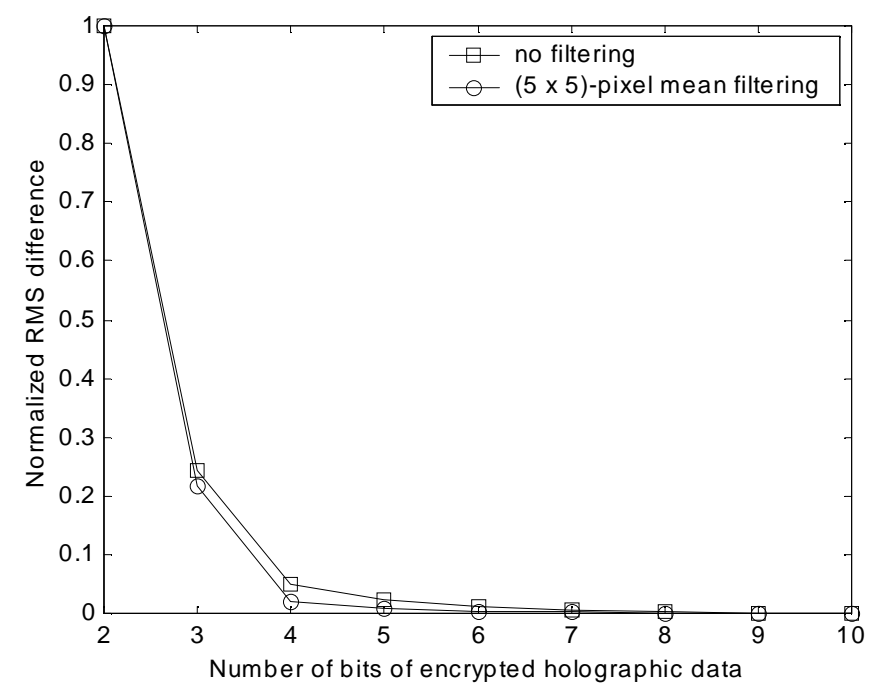

Figure 4. NRMS intensity difference in decrypted and reconstructed 3D bolt object plotted against quantization level

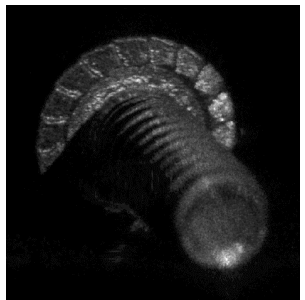

(a)

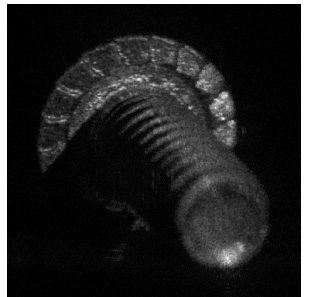

(b)

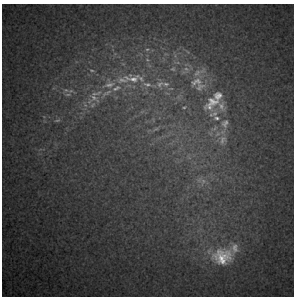

(c)

Figure 5. Decrypted and reconstructed bolt object (with $5 \times 5$ pixel mean filtering) from an encrypted digital hologram with the following resolution in each real and imaginary value: (a) 4 bits, (b) 3 bits, (c) 2 bits

in a real-world setting. Finally, both the networking software and compression software should be integrated so that meaningful conclusions can be drawn from the relative performance of the transmission and compression components of the system.

We have constructed an Internet-based digital hologram compression application in order to measure reliably and accurately the interaction between compression times and transmission times. This client-server application and associated compression algorithms were written with Java $^{\text {TM }}$ (Sun Microsystems, Inc). This allowed us to develop a platform-independent environment for experimentation over the Internet. Platform-independence ensures that the system supports any architecture that runs a Java virtual machine, and is suitable for heterogeneous environments (the server needs no knowledge of a client's computer architecture or operating system to communicate). As such, we ensure as much as possible the repeatability and relevance of our results for a wide range of Internet set-ups.

An overview of the operation of the networking application is shown in Fig. 6. Multiple clients, through their user interfaces, access the server and request particular views of 3D objects stored as digital holograms. The server responds by providing the appropriate window of pixels, and the clients reconstruct views of the $3 \mathrm{D}$ objects locally. To build the communication component for the client-server application we used Java's remote method invocation (RMI) facilities. RMI allows applications running on different machines to communicate with each other in an efficient and transparent manner. Our client-server system functions over any network (local, wide, wireless) that supports IP (Internet Protocol).

A request for a particular view of a particular 3D object is passed from the user interface, through the client, to the server. The server extracts the appropriate window from the digital hologram stored on disk, and formats it for transmission. Java is an object-oriented language. As such, digital holograms are stored as 


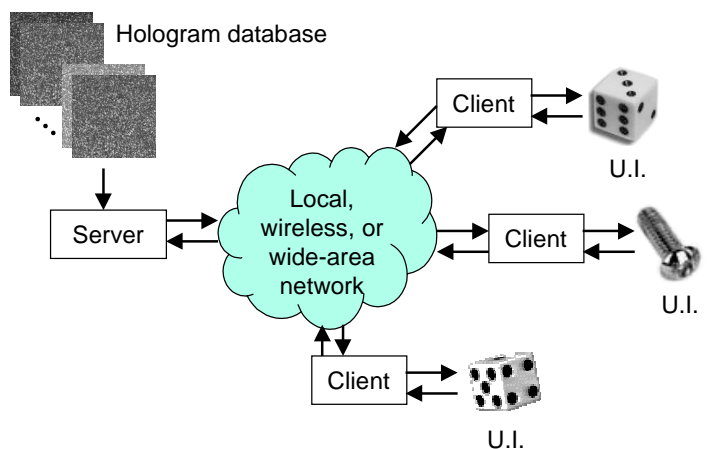

Figure 6. Illustration of the network-independent multiple-client system; U.I., user interface
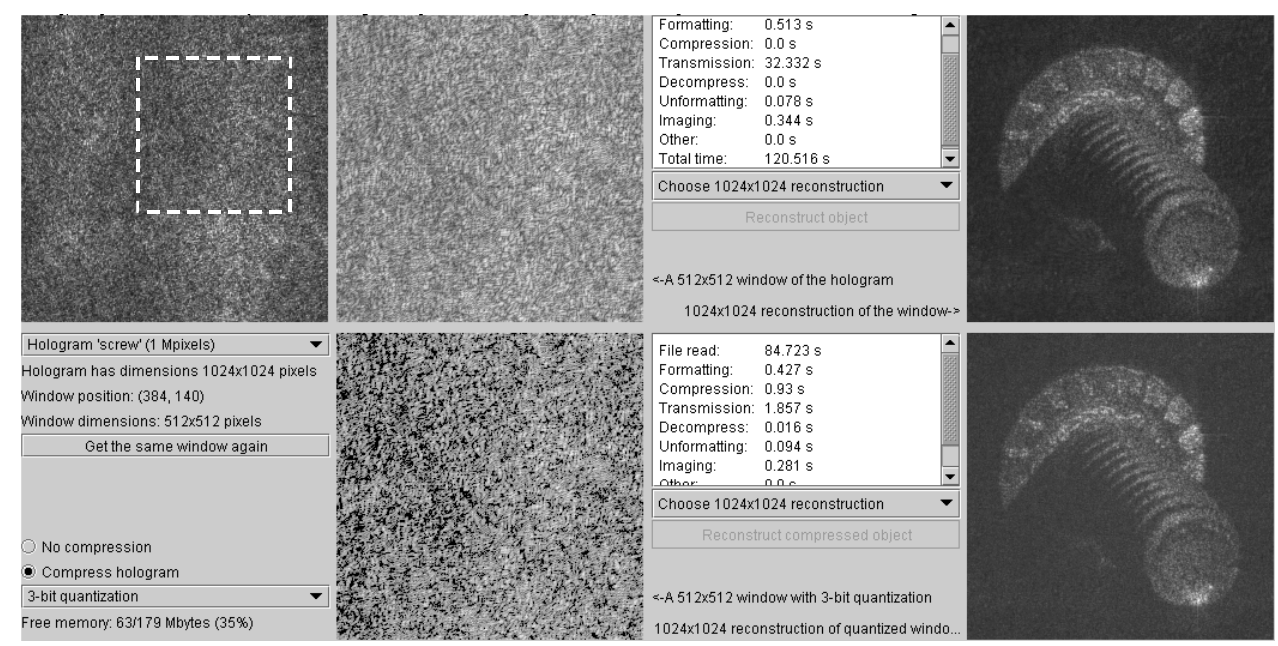

Figure 7. Screenshot of client-side of timings application. Top row (l to r): full digital hologram with window indicated, uncompressed digital hologram window data, uncompressed timings, and uncompressed reconstruction. Bottom row (l to r): control panel, compressed window, compressed timing information, and compressed reconstruction

generalized hologram objects, in a data format that allows efficient manipulation by specialized complex-valued digital hologram processing algorithms, and complete with the functionality to read/write them from/to disk, display them, and reconstruct their 3D objects. Formatting is required to streamline the hologram object for transmission, by removing all functionality and converting the field data into two compact 1D arrays of real and imaginary values. The number of quantization levels to be employed by the server is specified by the client.

The server collects timing information (time to read from disk, format, and compress) and transmits this with the hologram window. The server responds to the client through a dedicated communication channel that is set up through RMI, and closed immediately afterwards. The client, on receipt of the field data, recreates a hologram object that has the functionality to be propagated numerically and/or displayed as an intensity image at the user interface. Once again, timings are taken by the client for each of its operations. The client-side interface of the timing application is shown in Fig. 7. Our Internet-based digital hologram compression and timing application is accessible online at http://hologram.cs.may.ie .

\section{TIMING EXPERIMENTS}

For our experiments, the server was deployed on a Sun UltraSPARC 10 workstation at National University of Ireland, Maynooth, Ireland, and accessed by a client on a $2 \mathrm{GHz}$ Pentium 4 personal computer at University of Connecticut, USA. For several degrees of quantization, and several digital hologram window sizes, the client made requests to the server. Forty trials were recorded for each quantization and each window size. We conducted these trials overnight and at weekends during the period 2002.07.13 to 2002.07.25. The complete timing data for 
Table 1. Timings used to calculate speedup

\begin{tabular}{|c|c|c|c|c|c|c|c|c|}
\hline \multirow{2}{*}{$\begin{array}{l}\text { Win- } \\
\text { dow }\end{array}$} & \multirow{2}{*}{$\begin{array}{l}\text { Quant- } \\
\text { ization }^{a}\end{array}$} & \multicolumn{2}{|c|}{ Compress $^{b}$ (ms) } & \multicolumn{2}{|c|}{ Transmit $^{b}$ (ms) } & \multicolumn{2}{|c|}{ Decompress $^{b}(\mathrm{~ms})$} & \multirow{2}{*}{$\begin{array}{l}\text { Speed- } \\
\text { up }\end{array}$} \\
\hline & & $\mu$ & $\sigma$ & $\mu$ & $\sigma$ & $\mu$ & $\sigma$ & \\
\hline 512 & None & 0 & 0 & 36432.57 & 14699.4 & 0 & 0 & 1 \\
\hline 512 & 8 & 936.75 & 126.32 & 5882.85 & 1709.98 & 80.45 & 112.5 & 5.28 \\
\hline 512 & 7 & 909.25 & 95.77 & 4668.03 & 1346.59 & 45.7 & 10.76 & 6.48 \\
\hline 512 & 6 & 896.9 & 70.24 & 4181.5 & 1236.7 & 47.88 & 6.26 & 7.11 \\
\hline 512 & 5 & 899.93 & 76.77 & 3568.28 & 921.8 & 47.33 & 16 & 8.07 \\
\hline 512 & 4 & 880.5 & 111.12 & 3103.08 & 839.95 & 38.68 & 14.67 & 9.06 \\
\hline 512 & 3 & 868.2 & 77.98 & 2519.73 & 678.01 & 44.58 & 13.11 & 10.61 \\
\hline 512 & 2 & 781.75 & 43.1 & 2007.9 & 502.91 & 42.13 & 12.38 & 12.87 \\
\hline 64 & None & 0 & 0 & 1239.77 & 237.44 & 0 & 0 & 1 \\
\hline 64 & 8 & 10.8 & 2.38 & 380.6 & 105.36 & 0.5 & 3.13 & 3.16 \\
\hline 64 & 7 & 10.4 & 1.07 & 363.2 & 43.79 & 1 & 4.36 & 3.31 \\
\hline 64 & 6 & 11.1 & 2.71 & 358.95 & 103.54 & 1 & 4.36 & 3.34 \\
\hline 64 & 5 & 10.58 & 1.75 & 341.63 & 15.27 & 1.5 & 5.27 & 3.51 \\
\hline 64 & 4 & 11.35 & 3.04 & 339.43 & 15.93 & 1 & 4.36 & 3.52 \\
\hline 64 & 3 & 10.33 & 0.88 & 225.08 & 13.99 & 1.5 & 5.27 & 5.23 \\
\hline 64 & 2 & 10.38 & 1.58 & 220.63 & 17.62 & 0.01 & 0.01 & 5.37 \\
\hline
\end{tabular}

${ }^{a}$ Number of bits of resolution in each real and imaginary value.

${ }^{b} \mu$ and $\sigma$ calculated over 40 trials, rounded up to the nearest $0.01 \mathrm{~ms}$.

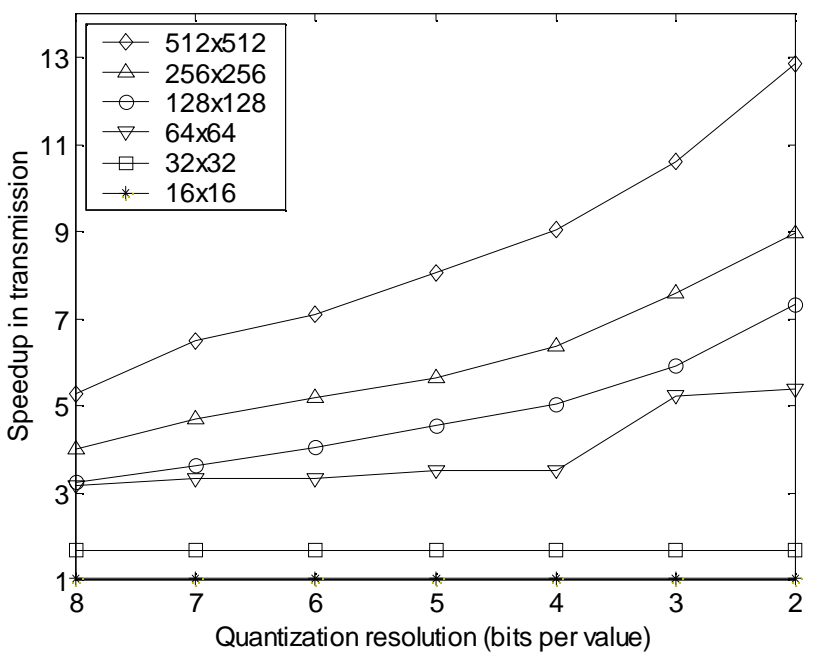

Figure 8. Speedup as a function of increasing compression, for various digital hologram window sizes

window sizes from $8 \times 8$ pixels to $512 \times 512$ pixels, and for quantizations from 8 bits to 2 bits was recorded. ${ }^{18}$ The $3 \mathrm{D}$ objects were not reconstructed numerically in the experiments; the complex-valued field was simply displayed as an intensity image.

A refined speedup metric is defined that avoids the bias effects that would be introduced by including file read, formatting, and imaging operations. These ancillary operations are independent of compression strategy and are significant in comparison to transmission time. The refined speedup $s^{\prime}$ is calculated from

$$
s^{\prime}=\frac{\overline{t_{\mathrm{u}}}}{\left(\bar{c}+\overline{t_{\mathrm{c}}}+\bar{d}\right)},
$$

where $t_{\mathrm{u}}$ and $t_{\mathrm{c}}$ are the uncompressed and compressed transmission times, respectively, $c$ and $d$ are the times to 
compress and decompress, respectively, and $\cdot$ denotes the mean of 40 trials. The timing data used to calculate speedup for two of the window sizes is given in Table 1. A plot of speedup against compression, for several window sizes, is shown in Fig. 8. For windows of size $64 \times 64$ pixels, or greater, there is significant speedup (over 2.5) for quantizations of 8 bits or lower. This speedup rises to over 12 for $512 \times 512$-pixel windows.

\section{CONCLUSION}

This paper outlined the results of combining quantization to the compression of encrypted digital holograms of 3D objects. The optical encryption technique, based on phase-shift digital holography, is suitable for secure 3D object storage and transmission applications. Lossless techniques, on their own, perform very poorly on encrypted (and unencrypted) digital holograms because of their white noise characteristics. Lossy quantization has been applied to good effect on the encrypted hologram data, and reductions to as few as 3 bits in each real and imaginary value have resulted in good quality decompressed and decrypted 3D object reconstructions. Not only does quantization perform significant compression itself, but it would also introduce redundancy into the bit stream to allow lossless techniques to subsequently perform more effectively. Finally, we use a speedup metric to validate that our compression techniques are viable for time-critical 3D imaging applications. Our Internet-based compression application and full timing results are accessible online. Our techniques are suitable for a range of secure $3 \mathrm{D}$ object storage and transmission applications.

\section{ACKNOWLEDGMENTS}

T.J. Naughton and J.B. McDonald acknowledge support from Enterprise Ireland and Science Foundation Ireland.

\section{REFERENCES}

1. J. W. Goodman and R. W. Lawrence, "Digital image formation from electronically detected holograms," Appl. Physics Lett. 11(2), 77-79, 1967.

2. M. A. Kronod, N. S. Merzlyakov, and L. P. Yaroslavskii, "Reconstruction of a hologram with a computer," Sov. Phys. Tech. Phys. 17, 333-334, 1972.

3. J. H. Bruning, D. R. Herriott, J. E. Gallagher, D. P. Rosenfeld, A. D. White, and D. J. Brangaccio, "Digital wavefront measuring interferometer for testing optical surfaces and lenses," Appl. Opt. 13, 2693-2703, 1974.

4. T.-C. Poon and A. Korpel, "Optical transfer function of an acousto-optic heterodyning image processor," Opt. Lett. 4, 317-319, 1979.

5. J. Schwider, B. Burow, K. E. Elsner, J. Grzanna, and R. Spolaczyk, "Digital wavefront measuring interferometry: some systematic error sources," Appl. Opt. 22, 3421-3432, 1983.

6. L. Onural and P. D. Scott, "Digital decoding of in-line holograms," Opt. Eng. 26, 1124-1132, 1987.

7. U. Schnars and W. P. O. Jüptner, "Direct recording of holograms by a CCD target and numerical reconstruction," Appl. Opt. 33, 179-181, 1994.

8. I. Yamaguchi and T. Zhang, "Phase-shifting digital holography," Opt. Lett. 22, 1268-1270, 1997.

9. B. Javidi and E. Tajahuerce, "Three-dimensional object recognition by use of digital holography," Opt. Lett. 25, 610-612, 2000.

10. Y. Frauel, E. Tajahuerce, M.-A. Castro, and B. Javidi, "Distortion-tolerant three-dimensional object recognition with digital holography," Appl. Opt. 40, 3887-3893, 2001.

11. O. Matoba and B. Javidi, "Optical retrieval of encrypted digital holograms for secure real-time display," Opt. Lett. 27, 321-323, 2002.

12. O. Matoba, T. J. Naughton, Y. Frauel, N. Bertaux, and B. Javidi, "Real-time three-dimensional object reconstruction by use of a phase-encoded digital hologram," Appl. Opt. 41, 6187-6192, 2002.

13. E. Tajahuerce, O. Matoba, and B. Javidi, "Shift-invariant three-dimensional object recognition by means of digital holography," Appl. Opt. 40, 3877-3886, 2001.

14. Y. Frauel and B. Javidi, "Neural network for three-dimensional object recognition based on digital holography," Opt. Lett. 26, 1478-1480, 2001. 
15. Y. Frauel, E. Tajahuerce, O. Matoba, A. Castro, and B. Javidi, "Comparison of passive ranging integral imaging and active imaging digital holography for three-dimensional object recognition," Appl. Opt. 43, 452-462, 2004.

16. E. Tajahuerce and B. Javidi, "Encrypting three-dimensional information with digital holography," Appl. Opt. 39, 6595-6601, 2000.

17. T. J. Naughton, Y. Frauel, B. Javidi, and E. Tajahuerce, "Compression of digital holograms for threedimensional object reconstruction and recognition," Appl. Opt. 41, 4124-4132, 2002.

18. T. J. Naughton, J. B. McDonald, and B. Javidi, "Efficient compression of Fresnel fields for Internet transmission of three-dimensional images," Appl. Opt. 42, 4758-4764, 2003.

19. T. J. Naughton and B. Javidi, "Compression of encrypted three-dimensional objects using digital holography," Opt. Eng. 43, 2233-2238, 2004.

20. B. Javidi and J. L. Horner, "Optical pattern recognition for validation and security verification," Opt. Eng. 33, 1752-1756, 1994.

21. P. Réfrégier and B. Javidi, "Optical image encryption based on input plane and Fourier plane random encoding," Opt. Lett. 20, 767-769, 1995.

22. S. Fukushima, T. Kurokawa, and Y. Sakai, "Image encipherment based on optical parallel processing using spatial light modulators," IEEE Photon. Technol. Lett. 3, 1133-1135, 1991.

23. M. Madjarova, M. Kakuta, M. Yamaguchi, and N. Ohyama, "Optical implementation of the stream cipher based on the irreversible cellular automata algorithm," Opt. Lett. 22, 1624-1626, 1997.

24. J. F. Heanue, M. C. Bashaw, and L. Hesselink, "Encrypted holographic data storage based on orthogonalphase-code multiplexing," Appl. Opt. 34, 6012-6015, 1995.

25. R. K. Wang, I. A. Watson, and C. R. Chatwin, "Random phase encoding for optical security," Opt. Eng. 35, 2464-2469, 1996.

26. L. G. Neto and Y. Sheng, "Optical implementation of image encryption using random phase encoding," Opt. Eng. 35(9), 2459-2463, 1996.

27. B. Javidi and E. Ahouzi, "Optical security system with Fourier plane encoding," Appl. Opt. 37, 6247-6255, 1998.

28. G. Unnikrishnan, J. Joseph, and K. Singh, "Optical encryption system that uses phase conjugation in a photorefractive crystal," Appl. Opt. 37, 8181-8186, 1998.

29. O. Matoba and B. Javidi, "Encrypted optical memory system using three-dimensional keys in the Fresnel domain," Opt. Lett. 24, 762-764, 1999.

30. P. C. Mogensen and J. Glückstad, "Phase-only optical encryption," Opt. Lett. 25, 566-568, 2000.

31. B. Javidi and T. Nomura, "Securing information by use of digital holography," Opt. Lett. 25, 28-30, 2000.

32. S. Lai and M. A. Neifeld, "Digital wavefront reconstruction and its application to image encryption," Opt. Comm. 178, 283-289, 2000.

33. E. Tajahuerce, O. Matoba, S. C. Verrall, and B. Javidi, "Optoelectronic information encryption with phaseshifting interferometry," Appl. Opt. 39, 2313-2320, 2000.

34. E. Tajahuerce, J. Lancis, B. Javidi, and P. Andrés, "Optical security and encryption with totally incoherent light," Opt. Lett. 26, 678-680, 2001.

35. B. Hennelly and J. T. Sheridan, "Optical image encryption by random shifting in fractional Fourier domains," Opt. Lett. 28, 269-271, 2003.

36. N. K. Nishchal, J. Joseph, and K. Singh, "Fully phase encryption using fractional Fourier transform," Opt. Eng. 42, 1583-1588, 2003.

37. J. W. Goodman and A. M. Silvestri, "Some effects of Fourier domain phase quantization," IBM J. Res. Develop. 14, 478-484, 1970.

38. W. J. Dallas and A. W. Lohmann, "Phase quantization in holograms - depth effects," Appl. Opt. 11, 192-194, 1972 .

39. T. Nomura, A. Okazaki, M. Kameda, Y. Morimoto, and B. Javidi, "Digital holographic data reconstruction with data compression," in Algorithms and Systems for Optical Information Processing V, B. Javidi and D. Psaltis, eds., Proceedings of SPIE vol. 4471, 235-242, (San Diego, California), 2001.

40. J. W. Goodman, Introduction to Fourier Optics, McGraw-Hill, New York, second ed., 1996.

41. H. J. Caulfield, ed., Handbook of Optical Holography, Academic Press, New York, 1979. 